\title{
THE CLASSIFICATION OF EXTENSIONS OF $C$ *-ALGEBRAS
}

\author{
BY JONATHAN ROSENBERG ${ }^{1}$ AND CLAUDE SCHOCHET ${ }^{1}$
}

1. G. G. Kasparov [9] recently showed how to construct a commutative semigroup $\operatorname{Ext}(A, B)$ out of "stable equivalence classes" of $C^{*}$-algebra extensions of the form

$$
0 \rightarrow B \otimes K \rightarrow E \rightarrow A \rightarrow 0,
$$

where $K=K\left(H^{\prime}\right)$ denotes the compact operators on a separable infinite-dimensional Hilbert space $H$. The addition operation comes from associating to (1.1) its "Busby invariant" [4, Theorem 4.3] $A \rightarrow 2(B)$, where $2(B)$ denotes the outer multiplier algebra of $B \otimes K$, and then using an injection of $Q(B) \oplus 2(B)$ into $Q(B)$. If $A$ is nuclear and $B$ has a countable approximate unit, $\operatorname{Ext}(A, B)$ is actually a group. The identity of the group corresponds to those extensions $\tau$ which are "stably split" (i.e., such that $\tau+\tau^{\prime}$ is split-see [4, Proposition 5.3]for some split extension $\tau^{\prime}$ ).

Study of groups which classify extensions of the form (1.1) was initiated by Brown, Douglas, and Fillmore (BDF) [3] in the case $B=\mathrm{C}$, the complex numbers. They showed that when $A=C(X), X$ a compact metric space, then unitary equivalence classes of unital "essential" extensions of the form (1.1) form a group $E \times t(A)$. Furthermore, Ext (_) generates a homology theory on compact metric spaces which satisfies Bott periodicity and other useful conditions. L. G. Brown [1] also proved the following Universal Coefficient Theorem (UCT): there is a natural short exact sequence

$$
0 \rightarrow \operatorname{Ext}\left(K^{0}(X), \mathbf{Z}\right) \rightarrow \operatorname{Ext}(C(X)) \stackrel{\gamma}{\rightarrow} \operatorname{Hom}\left(K^{1}(X), \mathbf{Z}\right) \rightarrow 0 .
$$

Here Ext and Hom denote $\operatorname{Ext}_{\mathbf{Z}}^{1}$ and $\operatorname{Hom}_{\mathbf{Z}}$, and $K^{*}$ is (complex) $K$-theory for compact spaces. Kahn, Kaminker, and Schochet [8] identified Ext $(C(X))$ for finite-dimensional compact metric spaces $X$ with the "Steenrod $K$-homology" of $X$ and reproved (1.2) in this case.

Our objective in this note is to outline a number of results concerning the Kasparov groups $\operatorname{Ext}(A, B)$ which are analogous to known information about the BDF theory. These results enable one to compute the groups with relatively mild restrictions on the $C^{*}$-algebras $A$ and $B$. This in turn should make it possible

Received by the editors June 23, 1980.

1980 Mathematics Subject Classification. Primary 46L05, 55N20, $18 \mathrm{G} 15$.

1 Research partially supported by the National Science Foundation 303-1690.

(C) 1981 American Mathematical Society $0002-9904 / 81 / 0000-0008 / \$ 02.50$ 
to analyze the way in which a wide variety of $C^{*}$-algebra extensions are put together, at least stably.

Our results were motivated by work of Brown (largely unpublished, but see [2]) extending the BDF results to the case where $A$ is no longer commutative or necessarily unital (but still $B=\mathrm{C}$ ). In this case $E x t(A)$ is a group, by results of Voiculescu, Choi-Effros, and Arveson, provided that $A$ is nuclear, and homotopy-invariance holds for a large subclass of such $A$ by work of Salinas and Pimsner-Popa-Voiculescu (PPV); see [6] for a detailed exposition.

2. Our first result concerns the relationship between Kasparov's $E \chi t(A, B)$ for $B$ abelian and certain other groups (unfortunately also denoted $E x t$, hence written here as $\left.E x t_{\mathrm{PPV}}\right)$ defined by PPV in [11] in a similar way from extensions of the form (1.1), but only using unital "homogeneous" extensions with $B=C(X), X$ a finite-dimensional compact metric.

THEOREM 2.1. Let $Y$ be a locally compact subset of some Euclidean space, and let $Y^{+}=Y \cup\{+\}$ be its one-point compactification. Let $A$ be a separable $C^{*}$-algebra, and $A^{+}$the algebra with unit adjoined. Then there is a natural isomorphism

$$
\operatorname{Ext} t_{\mathrm{PPV}}\left(Y^{+},+; A^{+}\right) \cong \operatorname{Ext}\left(A, C_{0}(Y)\right)
$$

\section{Corollary 2.2. Let $X$ and $Y$ be locally compact subsets of Euclidean} spaces. Then

$$
\operatorname{Ext}\left(C_{0}(X), C_{0}(Y)\right) \cong \widetilde{K}^{0}\left(Y^{+} \wedge F\left(X^{+}\right)\right),
$$

where $F\left(X^{+}\right)$is the function spectrum of $X^{+}$and $\widetilde{K}^{*}$ denotes reduced representable $K$-theory.

This follows from Theorem 2.1 and Schochet's calculation [12] of the PPV groups. Kasparov [9, Theorem 5] proved (2.2) when $X^{+}$and $Y^{+}$are finite CW-complexes.

Theorem 2.1 is not surprising for $Y$ compact, but was a surprise (to us, at least) in the noncompact case. The essence of the proof of (2.1) is to show (for $Y$ noncompact) that each map $A \rightarrow 2\left(C_{0}(Y)\right)$ is equivalent to one which "vanishes at infinity". For example, a certain quotient of the group $C^{*}$-algebra of the Heisenberg group, although shown by Voiculescu [14] to be nonsplit, is stably split by (2.2).

3. The following starting-point for computation of the Kasparov groups was obtained independently at about the same time by J. Cuntz [5]. ( $K_{*}$ denotes the Banach-algebra $K$-groups.) 
Theorem 3.1 (CF. [9, Theorem 5]). Let B be any $C^{*}$-algebra. Then there are natural isomorphisms

$$
E \times t(\mathrm{C}, B) \cong K_{1}(B) \text { and } E \times t\left(C_{0}(\mathrm{R}), B\right) \cong K_{0}(B) \text {. }
$$

The proof follows routinely from the direct construction of a unitary element in $2(B)$ whose class in $K_{1}(2(B))$ maps onto the class in $K_{0}(B)$ of any given projection in $B \otimes K$.

Our remaining results depend on deeper properties of the Kasparov groups. Let $E \times t_{1}=E x t, E \times t_{0}(A, B)=E \chi t\left(A \otimes C_{0}(\mathbf{R}), B\right)$. Henceforth assume that $A$ and $B$ are separable and $A$ is nuclear. $E \times t_{q}(A)=E \times t_{q}(A, \mathbf{C})(q=0,1)$ are the (weak) BDF groups.

TheOREM 3.2 (KASPAROV [9]). Ext $(A, B)$ is a homotopy-invariant bifunctor, covariant in $B$, contravariant in $A$, satisfying Bott periodicity, stability, and exactness axioms in each variable.

Let $\oplus$ denote the $c_{0}$-direct sum or "restricted direct product". The following analogue of the "infinite wedge axiom" in topology is similar to [3, Theorem 2.5].

Theorem 3.3. For any separable nuclear $C^{*}$-algebras $A_{1}, A_{2}, A_{3}, \ldots$, there is a natural isomorphism

$$
\text { Ext } *\left(\bigoplus_{n=1}^{\infty} A_{n}, B\right) \cong \prod_{n=1}^{\infty} \operatorname{Ext} *\left(A_{n}, B\right) .
$$

Corollary 3.4 (CF. Milnor [10], Brown [2]). Suppose that $A=$ $\stackrel{\lim }{\longrightarrow} A_{n}$ with $A_{n}$ nuclear. Then there is a natural short exact sequence

$$
0 \rightarrow \lim ^{1} \operatorname{Ext}\left(A_{n}, B\right) \rightarrow \operatorname{Ext}(A, B) \rightarrow \lim _{\leftarrow} \operatorname{Ext}\left(A_{n}, B\right) \rightarrow 0 .
$$

4. Theorem 4.1 (Künneth Theorem; CF. [12, Theorem 3]). Suppose that $A$ is an inductive limit of Type $I C^{*}$-algebras. Then the natural pairing

$$
\alpha: \operatorname{Ext}(A) \otimes K_{0}(B) \rightarrow \operatorname{Ext}(A, B)
$$

obtained from the obvious map $Q(C) \otimes_{\min } B \rightarrow 2(B)$ induces exact sequences $(p \in \mathbf{Z} / 2)$

$$
\begin{aligned}
0 & \rightarrow \bigoplus_{q \in \mathbf{Z} / 2} \operatorname{Ext}(A) \otimes K_{q+p}(B) \stackrel{\alpha}{\rightarrow} \operatorname{Ext}(A, B) \\
& \rightarrow \bigoplus_{q \in \mathbf{Z} / 2} \operatorname{Tor}\left(\operatorname{Ext}_{q+1}(A), K_{q+p}(B)\right) \rightarrow 0
\end{aligned}
$$

which are natural with respect to $A$ and $B$. 
Proof (SKetch). First suppose that $B$ is fixed with $K_{*}(B)$ free. Then (4.1) reduces to showing that $\alpha$ is an isomorphism. Using Theorem 3.2 and Corollary 3.4 , it is easy to see that

(a) if $A=\lim _{\rightarrow} A_{n}$ and (4.1) holds for all $\left(A_{n}, B\right)$, then it holds for $(A, B)$ and

(b) if $A$ contains a closed ideal $J$ and (4.1) holds for $(J, B)$ and $(A / J, B)$, then it holds for $(A, B)$.

Using structure theory for Type I $C^{*}$-algebras, one reduces to the case $A$ commutative, with, in fact, $\hat{A}$ a finite complex. Then $\alpha$ is a natural transformation of homology theories (on finite complexes) which is an isomorphism for 1-point spaces by (3.1), hence $\alpha$ is an isomorphism (by a spectral sequence argument).

The general case (with $K_{*}(B)$ arbitrary) is handled by the technique of a geometric projective resolution for $K_{*}(B)[13]$.

Theorem 4.2 (UCT). Suppose that $A$ is an inductive limit of Type I $C^{*}$. algebras. Then the natural map

$$
\gamma: \operatorname{Ext}(A, B) \rightarrow \bigoplus_{q \in \mathbf{Z} / 2} \operatorname{Hom}\left(K_{q}(A), K_{q+1}(B)\right)
$$

obtained from the connecting homomorphisms in the K-theory long exact sequence induces exact sequences $(p \in \mathbf{Z} / 2)$

$$
\begin{aligned}
& 0 \rightarrow \bigoplus_{q \in \mathbf{Z} / 2} \operatorname{Ext}\left(K_{q+1}(A), K_{q+p}(B)\right) \rightarrow \operatorname{Ext}(A, B) \\
& \stackrel{\gamma}{\rightarrow} \bigoplus_{q \in \mathrm{Z} / 2} \operatorname{Hom}\left(K_{q}(A), K_{q+p}(B)\right) \rightarrow 0
\end{aligned}
$$

which are natural in $A$ and $B$.

Corollary 4.3. Suppose that $A$ is an inductive limit of Type I $C^{*}$-algebras. Then there is a natural short exact sequence

$$
0 \rightarrow \operatorname{Ext}\left(K_{0}(A), \mathbf{Z}\right) \rightarrow \operatorname{Ext}(A) \stackrel{\gamma}{\rightarrow} \operatorname{Hom}\left(K_{1}(A), \mathbf{Z}\right) \rightarrow 0
$$

Proof. Set $B=C, p=1$ in (4.2).

Corollary 4.3 was established by Brown (unpublished) several years ago. Our attempt to understand his result and to give a more transparent proof led to some of our work on (4.2). If $A$ and $B$ are commutative then (4.2) may be approached via (2.2) and methods of algebraic topology). D. Handelman [7] proved (4.2) when $A$ and $B$ are AF algebras (at least with $B$ simple) and $p=1$; the UCT simplifies in this case since $K_{1}(A)=K_{1}(B)=0$. J. Cuntz [5] established (4.2) in the case where $A$ is an "O-algebra" associated with a Markov 
chain and $p=1$. Since such algebras are not inductive limits of Type I $C^{*}$-algebras, it seems that (4.1) and (4.2) ought to hold under weaker hypotheses on $A$, perhaps for all nuclear $C^{*}$-algebras.

Proof of (4.2) (SKeTCH). First suppose that $B$ is fixed with $K_{*}(B)$ divisible. Then (4.2) amounts to showing that $\gamma$ is an isomorphism, and the situation is handled as in the proof of the Künneth theorem. The general case (with $K_{*}(B)$ arbitrary) is handled by building a geometric injective resolution of $K_{*}(B)$.

The UCT has the following simple interpretation. Suppose $\tau$ is an extension of the form (1.1) with $A$ and $B$ satisfying the hypotheses of Theorem 4.2. Form the six-term $K$-theory exact sequence of (1.1). Suppose that (a) the boundary homomorphisms are zero and that (b) the two resulting short exact sequences of abelian groups split. Then $\tau$ is a stably split extension.

We would like to thank Larry Brown and Ed Effros for helpful conversations. We are particularly indebted to Brown for sharing with us some of his unpublished calculations and ideas.

Added in Proof. Since this was written, T. Fack and G. Skandalis have proved an analogue of A. Connes' Thom isomorphism theorem for the Kasparov groups. This makes it possible to enlarge the category of algebras in (4.1) and (4.2) to include crossed products by $\mathbf{R}$ and $\mathbf{Z}$.

\section{REFERENCES}

1. L. G. Town, Operator algebras and algebraic K-theory, Bull. Amer. Math. Soc. 81 (1975), 1119-1121.

2. Extensions and the structure of $C^{*}$-algebras, Istituto Nazionale di Alta Matematica, Sympos. Math. 20 (1976), 539-566.

3. L. G. Brown, R. G. Douglas, and P. A. Fillmore, Extensions of $C^{*}$-algebras and K-homology, Ann. of Math. (2) 105 (1977), 265-324.

4. R. S. Busby, Double centralizers and extensions of $C^{*}$-algebras, Trans. Amer. Math. Soc. 132 (1968), 79-99.

5. J. Cuntz, $A$ class of $C^{*}$-algebras and topological Markov chains. II: reducible Markov chains and the Ext-functor for $C^{*}$-algebras (preprint, 1980).

6. R. G. Douglas, $C^{*}$-algebra extensions and $K$-homology, Ann. of Math. Studies, no. 95 Princeton Univ. Press, Princeton, 1980.

7. D. Handelman, Extensions for AF algebras and dimension groups (preprint, 1979).

8. D. S. Kahn, J. Kaminker, and C. Schochet, Generalized homology theories on compact metric spaces, Michigan Math. J. 24 (1977), 203-224.

9. G. G. Kasparov, $K$-functors in the extension theory of $C^{*}$-algebras, Funkcional. Anal. i Priložen. 13 (1979), no. 4, 73-74. (Russian) $337-341$.

10. J. W. Milnor, On axiomatic homology theory, Pacific J. Math. 12 (1962),

11. M. Pimsner, S. Popa, and D. Voiculescu, Homogeneous $C^{*}$-extensions of $C(X) \otimes$ $K(H)$. I, J. Operator Theory 1 (1979), 55-108; II, ibid. (to appear).

12. C. Schochet, Homogeneous extensions of $C^{*}$-algebras and K-theory. I, Bull. Amer. Math. Soc. (N.S.) 3 (1980), 715-718. 
13. Topological methods for $C^{*}$-algebras. II: geometric resolutions and the Künneth formula (preprint, 1980).

14. D. Voiculescu, Remarks on the singular extension in the $C^{*}$-algebra of the Heisenberg group (preprint, 1979).

DEPARTMENT OF MATHEMATICS, UNIVERSITY OF PENNSYLVANIA, PHILADELPHIA, PENNSYLVANIA 19104 (ADDRESS OF JONATHAN ROSENBERG)

DEPARTMENT OF MATHEMATICS, WAYNE STATE UNIVERSITY, DETROIT, MICHIGAN 48202 (ADDRESS OF CLAUDE SCHOCHET) 